\title{
Complete Left-Invariant Affine Structures on Solvable Non-Unimodular Three-Dimensional Lie Groups
}

\section{Guediri $\mathrm{M}^{*}$ and Al-Balawi K}

Department of Mathematics, College of Science, King Saud University, Riyadh, Saudi Arabia

\author{
Abstract \\ In this paper, we shall use a method based on the theory of extensions of left-symmetric algebras to classify \\ complete left-invariant affine real structures on solvable non-unimodular three-dimensional Lie groups.
}

Keywords: Extensions of left-symmetric algebras; Left-invariant affine connections; Novikov algebras

\section{Introduction}

The notion of a left-symmetric algebra appeared for the first time in the work of Koszul [1] and Vinberg [2] concerning bounded homogeneous domains and convex homogeneous cones, respectively. Over the field of real numbers, left-symmetric algebras are of special interest because of their role in the differential geometry of affine manifolds (i.e. smooth manifolds with flat torsion-free affine connections), and in the representation theory of Lie groups $[3,4]$. In fact, for a given simply connected Lie group $G$ with Lie algebra $\mathcal{G}$, the left-invariant affine structures on $\mathcal{G}$ are in one-to-one correspondence with the left-symmetric structures on $G$ compatible with the Lie structure [5].

On the other hand, it is well known that there is a one-to-one correspondence between left-invariant affine structures on a Lie group $G$ and locally simply transitive affine actions of $G$ on an n-dimensional real vector space $V$ [5]. The classification of left-invariant affine structures on a given Lie group $G$ is then reduced to the classification of compatible left-symmetric products on the Lie algebra $\mathcal{G}$ of G. It has been proved [6] that a simply connected Lie group $G$ which acts simply transitively on $\mathbb{R}^{n}$ by affine transformations is necessarily solvable. Since a few years, there has been a growing interest in the study of simply transitive affine actions of Lie groups on $\mathbb{R}^{n}$. This interest is mostly due to the example of Benoist [7], who constructed a simply connected nilpotent Lie group not admitting any locally simply transitive affine action on $\mathbb{R}^{n}$. This example provided a negative answer to the following question of Milnor [3]. Does any simply connected solvable Lie group admit a simply transitive affine action on $\mathbb{R}^{n}$ ?

From another point of view, there is also the question of classifying all simply transitive affine actions of a given solvable Lie group $\mathrm{G}$ admitting such an action. This question, even in the abelian case $G=\mathbb{R}^{k}$, seems to be very hard. When $G$ is nilpotent, the classification has been completely achieved up to dimension four $[8,9]$.

Recently, a method based on the theory of extensions of leftsymmetric algebras has been proposed [10] to classify complete leftinvariant affine real structures on a given solvable Lie group of low dimension. Since the classification in the case of solvable unimodular Lie groups of dimension three was obtained [8], we will use that method to carry out in this paper the classification of complete left-invariant affine structures on three-dimensional solvable non-unimodular Lie groups.

The paper is organized as follows. In section 2, we will briefly recall some necessary definitions and basic results on left-symmetric algebras and their extensions. In section 3, using the classification of the threedimensional complex simple left-symmetric algebras given [11] and a result [12], we shall first show that any complete real left-symmetric algebra $A_{3}$ of dimension 3 whose Lie algebra is solvable and nonunimodular is not simple. Therefore, we can get $A_{3}$ as an extension of complete left-symmetric algebras. By using the Lie group exponential maps, we shall deduce the classification of all complete left-invariant affine structures on solvable non-unimodular Lie groups of dimension 3 in terms of simply transitive actions of subgroups of the affine group $\operatorname{Aff}\left(\mathbb{R}^{3}\right)=G L\left(\mathbb{R}^{3}\right) \times \mathbb{R}^{3}$ (see Theorem 13).

Throughout this paper, all considered vector spaces, Lie algebras, and left-symmetric algebras are supposed to be over the field $\mathbb{R}$. We shall also suppose that all considered Lie groups are simply connected.

\section{Left-symmetric Algebras and their Extensions}

Let $A$ be a finite-dimensional vector space over $\mathbb{R}$. A left-symmetric product on $\mathrm{A}$ is a bilinear product that we denote by $x \cdot y$ satisfying

$$
(x \cdot y) \cdot z-(y \cdot x) \cdot z=x \cdot(y \cdot z)-y \cdot(x \cdot z),
$$

for all $[x, y]=x \cdot y-y \cdot x$. In this case, $A$ together with a left-symmetric product is called left-symmetric algebra.

Now if $A$ is a left-symmetric algebra, then the commutator

$$
[x, y]=x \cdot y-y \cdot x
$$

defines a structure of Lie algebra on $A$, called the associated Lie algebra. On the other hand, if $\mathcal{G}$ is a Lie algebra with a left-symmetric product satisfying (2), then we say that this left-symmetric structure is compatible with the Lie structure on $\mathcal{G}$.

Let $G$ be a simply connected Lie group with a left-invariant affine connection $\nabla$. Define a product $\bullet$ on the Lie algebra $\mathcal{G}$ of $G$ by

$$
x \cdot y=\nabla_{x} y,
$$

for all $x, y \in \mathcal{G}$. Then, the flat and torsion-free conditions on $\nabla$

*Corresponding author: Guediri M, Department of Mathematics, College of Science, King Saud University 2455, Riyadh 11451, Saudi Arabia, Tel: 96611467 0000; E-mail: mguediri@ksu.edu.sa

Received February 18, 2014; Accepted June 29, 2015; Published July 07, 2015

Citation: Guediri M, Al-Balawi K (2015) Complete Left-Invariant Affine Structures on Solvable Non-Unimodular Three-Dimensional Lie Groups. J Generalized Lie Theory Appl 9: 222. doi:10.4172/1736-4337.1000222

Copyright: $\odot 2015$ Guediri M, et al. This is an open-access article distributed unde the terms of the Creative Commons Attribution License, which permits unrestricted use, distribution, and reproduction in any medium, provided the original author and source are credited. 
Citation: Guediri M, Al-Balawi K (2015) Complete Left-Invariant Affine Structures on Solvable Non-Unimodular Three-Dimensional Lie Groups. J Generalized Lie Theory Appl 9: 222. doi:10.4172/1736-4337.1000222

Page 2 of 11

correspond to conditions (1) and (2), respectively.

Conversely, If $G$ is a simply connected Lie group with Lie algebra $\mathcal{G}$ and $x \cdot y$ denotes a left-symmetric product on $\mathcal{G}$ compatible with the Lie bracket, then the left-invariant connection given by $\nabla_{x} y=x \cdot y$ defines a left-invariant affine structure $\nabla$ on $\mathcal{G}$. We deduce that if $G$ is a simply connected Lie group with Lie algebra $\mathcal{G}$, then the study of left-invariant affine structures on $G$ is equivalent to the study of leftsymmetric structures on $G$ compatible with the Lie structure.

Let $A$ be a left-symmetric algebra whose associated Lie algebra is $\mathcal{G}$, and let $\mathrm{L}_{\mathrm{x}}$ and $\mathrm{R}_{\mathrm{x}}$ denote the left and right multiplications, respectively i.e. $L_{x} y=x \cdot y$ and $R_{x} y=x \cdot y$. The identity in (1) is now equivalent to the formula

$$
\left[L_{x}, L_{y}\right]=L_{[x, y]} \text {, for all } \mathrm{x}, \mathrm{y} \in \mathrm{A} \text {, }
$$

or, in other words, the linear map $L: \mathcal{G} \rightarrow E n d(A)$ is a representation of Lie algebras.

If a left-symmetric algebra $A$ has no proper two-sided ideal and it is not the zero algebra of dimension 1 , then $A$ is called simple. $A$ is called semi simple, if it is a direct sum of simple left-symmetric algebras.

We say that $A$ is complete if $R_{x}$ is a nilpotent operator for all $x \in \mathrm{A}$. It turns out that, for a given simply connected Lie group $G$ with Lie algebra $\mathcal{G}$, the complete left-invariant affine structures on $G$ are in one-to-one correspondence with the complete left-symmetric structures on $\mathcal{G}$ compatible with the Lie structure. It is also known that an $n$-dimensional simply connected Lie group admits a complete leftinvariant affine structure if and only if it acts simply transitively on $\mathbb{R}^{n}$ by affine transformations [9]. A simply connected Lie group which is acting simply transitively on $\mathbb{R}^{n}$ by affine transformations must be solvable according to [6]. It is well known that not every solvable (even nilpotent) Lie group can admit an affine structure [7].

We say that $A$ is Novikov algebra if it satisfies the identity

$$
(x \cdot y) \cdot z=(x \cdot z) \cdot y, \quad \text { for all } \mathrm{x}, \mathrm{y} \in \mathrm{A} \text {. }
$$

In terms of left and right multiplications, (3) is equivalent to the formula

$$
\left[R_{x}, R_{y}\right]=0 \text {, for all } \mathrm{x}, \mathrm{y} \in \mathrm{A} \text {. }
$$

The left-symmetric algebra $A$ is called derivation algebra if it satisfies the identity

$$
(x \cdot y) \cdot z=(z \cdot y) \text {, for all } \mathrm{x}, \mathrm{y}, \mathrm{z} \in \mathrm{A}
$$

or, equivalently, all left and right multiplications $L_{x}$ and $R_{x}$ are derivations of $g$.

Recall that a Lie algebra $\tilde{\mathcal{G}}$ is an extension of the Lie algebra $\mathcal{G}$ by the Lie algebra $A$ if there exists a short exact sequence of Lie algebras

$$
0 \rightarrow \mathcal{A} \stackrel{i}{\rightarrow} \tilde{\mathcal{G}} \stackrel{\pi}{\rightarrow} \mathcal{G} \rightarrow 0 .
$$

In other words, $A$ is an ideal of $\tilde{\mathcal{G}}$ such that $\tilde{\mathcal{G}} / A \cong \mathcal{G}$.

For $(x, a)$ and $(y, b)$ in $\tilde{\mathcal{G}} \cong \mathcal{G} \oplus A$, the extended Lie bracket is given by

$$
[(x, a),(y, b)]=([x, y],[a, b]+\phi(x) b-\phi(y) a+\omega(x, y)),
$$

where $\phi: \mathcal{G} \rightarrow \operatorname{Der}(A)$ is a linear map and $w: \mathcal{G} \times \mathcal{G} \rightarrow A$ is an alternating bilinear map such that

$$
[\phi(x), \phi(y)]=\phi([x, y])+a d_{\omega(x, y)},
$$

and

$\omega([x, y], z)-\omega(x,[y, z])+\omega(y,[x, z])=\phi(x) \omega(y, z)+\phi(y) \omega(z, x)+\phi(z) \omega(x, y)$.

Note here that if $A$ is abelian, then $\omega$ is a 2-cocycle $[13,14]$.

Now we shall briefly discuss the problem of extension of a left-symmetric algebra by another left-symmetric algebra. To our knowledge, the notion of extensions of left-symmetric algebras has been considered for the first time in [9], to which we refer the reader for more details [15]

Suppose that a vector space extension of a left-symmetric algebra $A$ by another left-symmetric algebra $E$ is given. We want to define a leftsymmetric structure on $\tilde{A}$ in terms of the left-symmetric structures given on $A$ and $E$. In other words, we want to define a left-symmetric product on $\tilde{A}$ for which $E$ becomes a two-sided ideal in $\tilde{A}$ such that $\tilde{A} / \mathrm{E} \cong A$; or equivalently,

$0 \rightarrow E \rightarrow \tilde{A} \rightarrow A \rightarrow 0$

Becomes a short exact sequence of left-symmetric algebras.

Theorem 1:There exists a left-symmetric structure on $\tilde{A}$ extending a left-symmetric algebra $A$ by a left-symmetric algebra $E$ if and only if there exist two linear maps $\lambda, \rho: A \rightarrow \operatorname{End}(\mathrm{E})$ and a bilinear map $g: A \times A \rightarrow E$ suct that for all $x, y, z \in A$ and $a, b \in E$, the following conditions are satisfied [9].

$$
\begin{array}{ll}
1 & \lambda_{x}(a \cdot b)=\lambda_{x}(a) \cdot b+a \cdot \lambda_{x}(b)-\rho_{x}(a) \cdot b, \\
2 & \rho_{x}([a, b])=a \cdot \rho_{x}(b)-b \cdot \rho_{x}(a), \\
3 & {\left[\lambda_{x}, \lambda_{y}\right]-\lambda_{[x, y]}=L_{g(x, y)-g(y, x)},} \\
4 & {\left[\lambda_{x}, \rho_{y}\right]+\rho_{y}{ }^{\circ} \rho_{x}-\rho_{x \cdot y}=R_{g(x, y)}} \\
5 & g(x, y \cdot z)-g(y, x \cdot z)+\lambda_{x}(g(y, z))-\lambda_{y}(g(x, z))-g([x, y], z) \\
- & \rho_{z}(g(x, y)-g(y, x))=0 .
\end{array}
$$

If the conditions of the above theorem are fulfilled, then the extended left-symmetric product on $A \cong A \times E$ is given by

$$
(x, a) \cdot(y, b)=\left(x \cdot y, a \cdot b+\lambda_{x}(b)+\rho_{y}(a)+g(x, y)\right) .
$$

It is remarkable that if the left-symmetric product of $\mathrm{E}$ is trivial, then the conditions of the above theorem simplify to the following three conditions:

(i) $\left[\lambda_{x}, \lambda_{y}\right]=\lambda_{[x, y \%]}$, i.e. $\lambda$ is a representation of Lie algebras,

(ii) $\left[\lambda_{x}, \rho_{y}\right]=\rho_{x \cdot y}-\rho_{y}{ }^{\circ} \rho_{x}$.

(iii) $g(x, y \cdot z)-g(y, x \cdot z)+\lambda_{x}(g(y, z))-\lambda_{y}(g(x, z))-g([x, y], z)$

$-\rho_{z}(g(x, y)-g(y, x))=0$.

In this case, $E$ becomes an $A$-bimodule and the extended product given in (5) simplifies too. Recall that if $K$ is a left-symmetric algebra and $V$ is a vector space, then we say that $V$ is a $K$-bimodule if there exist two linear maps $\lambda, \rho: K \rightarrow$ End $(V)$ which satisfy the conditions (i) and (ii) stated above.

Let $K$ be a left-symmetric algebra, and suppose that a $K$-bimodule $V$ is known. We denote by $L^{p}(K, V)$ the space of all $p$-linear maps from $K$ to $V$, and we define two co-boundary operators $\delta_{1}: L^{1}(K, V) \rightarrow L^{2}(K, V)$ and $\delta_{2}: L^{2}(K, V) \rightarrow L^{3}(K, V)$ as follows:

For a linear map $h \in L^{1}(K, V)$ we set 
Citation: Guediri M, Al-Balawi K (2015) Complete Left-Invariant Affine Structures on Solvable Non-Unimodular Three-Dimensional Lie Groups. J Generalized Lie Theory Appl 9: 222. doi:10.4172/1736-4337.1000222

Page 3 of 11

$$
\delta_{1} h(x, y)=\rho_{y}(h(x))+\lambda_{x}(h(y))-h(x \cdot y),
$$

and for a bilinear map $g \in L^{2}(K, V)$ we set

$\delta_{2} g(x, y, z)=g(x, y \cdot z)-g(y, x \cdot z)+\lambda_{x}(g(y, z))-\lambda_{y}(g(x, z))-g([x, y], z)-\rho_{z}(g(x, y)-g(y, x))$

where $\lambda$ and $\rho$ are linear maps $\lambda, \rho: K \rightarrow \operatorname{End}(V)$.

It is straightforward to check that $\delta_{2} \mathrm{o} \delta_{1}=0$. Therefore, if we set $Z_{\lambda, \rho}^{2}(K, V)=\operatorname{ker} \delta_{2}$ and $B_{\lambda, \rho}(K, V)$ Im ${ }_{1}$, we can define a notion of second co-homology for the actions $\lambda$ and $\rho$ by simply setting $H_{\lambda, \rho}^{2}(K, V)=Z_{\lambda, \rho}^{2}(K, V) / B_{\lambda, \rho}^{2}(K, V)$. As in the case of Lie algebras, we can prove the following [9].

Proposition 2: For given linear maps $\lambda, \rho: K \rightarrow \operatorname{End}(V)$, the equivalent classes of extensions

$$
0 \rightarrow V \rightarrow A \rightarrow K \rightarrow 0
$$

of $K$ by $V$ are in one-to - one correspondence with the elements of the second co-homology group $H_{\lambda, \rho}^{2}(K, V)$.

A left-symmetric algebras extension

$$
0 \rightarrow E \stackrel{i}{\rightarrow} \tilde{A} \stackrel{\pi}{\rightarrow} A \rightarrow 0
$$

is called central if and only if $i(E) \subseteq C(\tilde{A})$ where

$$
C(\tilde{A})=\{x \in \tilde{A}: x \cdot y=y \cdot x=0\}
$$

is the center of $\tilde{A}$. In particular, the extension is central whenever $E$ is a trivial $A$-bimodule (i.e. $\lambda=\rho=0$ ). We say that the extension is exact if and only if $i(E)=C(\tilde{A})$. It is easy to verify [9] that the extension is exact if and only if $I_{[\mathrm{g}]} 0$, where

$$
I_{[g]}=\{x \in A: x \cdot y=y \cdot x=0 \text { and } g(x, y)=g(y, x)=0 \text { for all } \mathrm{y} \in \mathrm{A}\}
$$

We observe that $I_{[\mathrm{g}]}$ is depends only on the co-homology class of $\mathrm{g}$, that is $I_{[\mathrm{g}]}$ is well defined. In case $E$ is a trivial $A$ - bimodule, we denote the central extension corresponding to the class $[g] \in H^{2}(A, E) b y(\tilde{A},[g])$.

Let $(\tilde{A},[g])$ and $\left(\tilde{A},\left[g^{\prime}\right]\right)$ be two central extensions of $A$ by $E$, $\mu \in \operatorname{Aut}(E)=G L(E)$ and $\eta \in A u t(A)$, where Aut (E) and Aut (A) are the groups of left-symmetric automorphisms of $E$ and $K$, respectively. It is clear that if, $h \in L^{1}(A, E)$, then the linear mapping $\psi: \tilde{A} \rightarrow \tilde{A}^{\prime}$ defined by

$$
\psi(x, a)=(\eta(x), \mu(a)+h(x))
$$

is an isomorphism provided

$$
g^{\prime}(\eta(x), \eta(y))=\mu(g(x, y))+\delta_{1} h(x, y) \text { forall }(x, y) \in A \times A \text {, i.e., } \eta^{*}\left[\mathrm{~g}^{\prime}\right]=\mu_{*}[g] \text {. }
$$

This allows us to define an action of the group $G=A u t(E) \times A u t(A)$ on $H^{2}(A, E)$ by setting

$$
(\mu, \eta) \cdot[g]=\mu_{*} \eta^{*}[g]
$$

or equivalently, $(\mu, \eta) \cdot g(x, y)=\mu(g(\eta(x), \eta(y)))$ for all $x, y \in A$.

Denoting the set of all exact central extensions of $A$ by $E$ by

$$
H_{e x}^{2}(A, E)=\left\{[g] \in H^{2}(A, E): I_{[g]}=0\right\}
$$

and the orbit of $[g]$ by $G_{[g]}$ it turns out that the following result is valid [9].

Proposition 3: Let $[g]$ and $\left[g^{\prime}\right]$ be two classes in $H_{e x}^{2}(A, E)$. Then, the central extensions $(\tilde{A},[g])$ and $\left(\tilde{A}^{\prime},\left[g^{\prime}\right]\right)$ are isomorphic if and only if $G_{[g]}=G\left[g^{\prime}\right]$. In other words, the classification of the exact central extensions of $A$ by $E$ is, up to left-symmetric isomorphism the orbit space of $H_{e x}^{2}(A, E)$ under the natural action of $G=A u t(E) \times A u t(A)$.

We close this section by the following important result [15].

Proposition 4: Let $0 \rightarrow I \rightarrow A \% \rightarrow J \rightarrow 0$ be an exact sequence of leftsymmetric algebras such that $A$ is complete then I and J are complete

Proof: Let $A$ be a complete left-symmetric algebra. Then $R_{x}$ is nilpotent for all $x \in A$. Since $\mathrm{J}$ is an ideal of $A$, then $R_{x}$ is nilpotent for all $x \in I$, that is $I$ is complete. On the other hand, Since $J \cong A / I$, we can define for $x \in A,\left.R_{x}\right|_{J}: J \rightarrow J$, by $\left.R_{x}\right|_{J}(\bar{y})=R_{x} y+I$ for all $y \in A$, $\bar{y}=y+I$. Since for all $y_{1}, y_{2} \in A$ such that $y_{l}+I=y_{2}+I$ there exists $z \in I$ so that $y_{2}=y_{l}+z$, and

$$
\begin{aligned}
& R_{x}\left(y_{2}+I\right)=R_{x} y_{2}+I \\
& =R_{x}\left(y_{l}+z\right)+I \\
& =R_{x} y_{l}+R_{x} z+I \\
& =R_{x} y_{l}+I \\
& =R_{x}\left(y_{l}+I\right)
\end{aligned}
$$

then, $\left.R_{x}\right|_{J}$ is well defined. We also have, for all $x, y \in A$, that

$$
\begin{aligned}
R_{\bar{x}} \bar{y} & =(y+I) \cdot(x+I) \\
& =y \cdot x+I \\
& =R_{x} y+I \\
& =R_{x} \bar{y}
\end{aligned}
$$

Thus, to prove that $J$ is complete, it is enough to prove that $\left.R_{x}\right|_{J}$ is nilpotent for all $x \in A$. Since $\mathrm{R}_{\mathrm{x}}$ is nilpotent, then $R_{x}^{k}=0$ for some $k \in \mathbb{N}$. This implies that

$$
R_{x}^{k}(y)+I=I=\overline{0}
$$

for all $y \in A$ Hence, $R_{x}^{k}(\bar{y})=0$ for all $\bar{y} \in J$, that is $\left.R_{x}\right|_{J}$ is nilpotent for all $x \in A$, and hence $J$ is complete.

\section{Complete Left-Symmetric Structures on Solvable Non- Unimodular Lie Algebras of Dimension 3}

Recall that a lie algebra $\mathcal{G}$ is unimodular if and only if $\operatorname{tr}\left(a d_{x}\right)=0$ for all $x \in \mathcal{G}$. The classification of solvable non unimodular Lie algebras of dimension 3 can be found [16].

Lemma 5: Let $\mathrm{g}$ be solvable non-unimodular Lie algebra of dimension 3. Then there is a basis $\left\{e_{p}, e_{2}, e_{3}\right\}$ of $\mathcal{G}$ so that

$$
\begin{aligned}
& {\left[e_{1}, e_{2}\right]=\alpha e_{2}+\beta e_{3}} \\
& {\left[e_{1}, e_{3}\right]=\gamma e_{2}+(2-\alpha) e_{3}}
\end{aligned}
$$

If we exclude the case where $D$ is the identity matrix then the determinant $\operatorname{det} D=\alpha(2-\alpha)-\beta \gamma$ provides a complete isomorphism invariant for this Lie algebra.

According to this result, we can, by simple computations, find that there are five possibilities for D:

$$
\begin{aligned}
& D \cong\left(\begin{array}{ll}
0 & 0 \\
0 & 1
\end{array}\right), \quad D \cong\left(\begin{array}{ll}
1 & 0 \\
0 & 1
\end{array}\right), \quad D \cong\left(\begin{array}{ll}
1 & 0 \\
1 & 1
\end{array}\right), \\
& D \cong\left(\begin{array}{ll}
0 & 0 \\
0 & \mu
\end{array}\right), \text { where } 0<|\mu|<1 \text { or } D \cong\left(\begin{array}{ll}
0 & -\varsigma \\
\varsigma & 1
\end{array}\right) \text { where } \varsigma>0
\end{aligned}
$$

This implies that any solvable non-unimodular Lie algebra of 
Citation: Guediri M, Al-Balawi K (2015) Complete Left-Invariant Affine Structures on Solvable Non-Unimodular Three-Dimensional Lie Groups. J Generalized Lie Theory Appl 9: 222. doi:10.4172/1736-4337.1000222

Page 4 of 11

dimension 3 is isomorphic to one and only one of the following Lie algebras

$$
\begin{aligned}
& \mathcal{G}_{3,1}:\left[e_{1}, e_{2}\right]=e_{2} \\
& \mathcal{G}_{3,2}:\left[e_{1}, e_{2}\right]=e_{2},\left[e_{1}, e_{3}\right]=e_{3} \\
& \mathcal{G}_{3,3}:\left[e_{1}, e_{2}\right]=e_{2}+e_{3},\left[e_{1}, e_{3}\right]=e_{3} \\
& \mathcal{G}_{3,4}^{\mu}:\left[e_{1}, e_{2}\right]=e_{2},\left[e_{1}, e_{3}\right]=\mu e_{3}, 0<|\mu|<1 \\
& \mathcal{G}_{3,5}^{\zeta}:\left[e_{1}, e_{2}\right]=e_{2}+\zeta e_{3},\left[e_{1}, e_{3}\right]=-\zeta e_{2}+e_{3}, \zeta>0
\end{aligned}
$$

Now let $\mathcal{G}$ be real solvable non-unimodular Lie algebra of dimension 3. Let $A_{3}$ be a complete left- symmetric algebra whose associated Lie algebra is $\mathcal{G}$.

We shall first recall the following result from [12].

Lemma 6: Only the complex sim le left-symmetric algebras and even-dimensional complex semisim le left-symmetric algebras may have simple real forms, where a real form of a complex left-symmetric algebra $A$ is sub algebra $A_{0}$ of $A^{\mathbb{R}}$ such that $A_{0}^{\mathbb{C}}=$ A. Here $A^{\mathbb{R}}$ is $A$ regarded as a real left-symmetric algebra.

Now, we can prove the following

Proposition 7: $A_{3}$ is not simple. In other words, any complete left-symmetric structure on a solvable non-unimodular Lie algebra of dimension 3 is not simple.

Proof: Assume to the contrary that $A_{3}$ is simple. Then, Lemma 6 shows that complexification $A_{3}^{\mathbb{C}}$ of $A_{3}$ is simple as the dimension of $A_{3}^{\mathbb{C}}$ is odd. We can now apply Corollary 4.2 in [11] to deduce that $A_{3}^{\mathbb{C}}$ is isomorphic to the complex left-symmetric algebra $A_{1}^{-1}$ having a basis $\left\{e_{1}, e_{2}, e_{3}\right\}$ such that the only non-trivial products are

$$
\begin{aligned}
& e_{1} \cdot e_{2}=e_{2}, \\
& e_{1} \cdot e_{3}=-e_{3}, \\
& e_{2} \cdot e_{3}=e_{3} \cdot e_{2}=e_{1} .
\end{aligned}
$$

Thus, the complex lie algebra $\mathcal{G}_{3}$ associated to $A_{3}^{\mathbb{C}} \cong A_{1}^{-1}$ is unimodular and hence $\mathcal{G}$ must be unimodular. This contradiction shows that $A_{3}$ is not simple

Before returning to the left-symmetric algebra $A_{3}$, we need to state the following facts without proofs.

Lemma 8: Let $A$ be a left-symmetric algebra with associated Lie algebra $\mathcal{G}$ and $R$ a two-sided ideal in $A$. Then the lie algebra $R$ associated to $R$ is an ideal in $\mathcal{G}$

Lemma 9: Let $\mathcal{G}$ be solvable non-unimodular Lie algebra of dimension 3 and let $\mathcal{I}$ be a proper ideal of $\mathcal{G}$. Then $\mathcal{I}$ is isomorphic to $\mathbb{R} \mathbb{R}^{2}$, aff $(\mathbb{R})=\left\langle e_{1}, e_{2}:\left[e_{1}, e_{2}\right]=e_{2}\right\rangle$.

By Proposition 7, $A_{3}$ is not simple and hence it has a proper twosided ideal $I$, so we get a short exact sequence of left-symmetric algebras

$$
0 \rightarrow I \stackrel{i}{\rightarrow} A_{3} \stackrel{\pi}{\rightarrow} J \rightarrow 0
$$

If $\mathcal{I}$ is the Lie sub algebra associated to $I$ then, by Lemma $8, \mathcal{I}$ is an ideal in $\mathcal{G}$. From Lemma 9 it follows that there are three cases to be considered according to weather $\mathcal{I}$ is isomorphic to $\mathbb{R}, \mathbb{R}^{2}$, or off $(\mathbb{R})$.

Case $1: \mathcal{I} \cong \mathbb{R}$.

In this case, the short exact sequence (8) becomes

$$
0 \rightarrow \mathbb{R}_{0} \rightarrow A_{3} \rightarrow I_{2} \rightarrow 0
$$

where $\mathrm{I}_{2}$ is a complete left-symmetric algebra of dimension 2 and $\mathbb{R}_{0}$ is $\mathbb{R}$ with the trivial product. At the Lie algebra level, we have a short exact sequence of Lie algebras of the form

$$
0 \rightarrow \mathbb{R} \rightarrow \tilde{\mathcal{G}} \rightarrow \mathcal{H}_{2} \rightarrow 0
$$

where $\mathcal{H}_{2}$ denotes the associated Lie algebra of $\mathrm{I}_{2}$ and $\tilde{\mathcal{G}}$ is an extension of $\mathcal{H}_{2}$ by $\mathbb{R}$.

Since $\mathcal{H}_{2}$ is of dimension 2, then $\mathcal{H}_{2}$ is either isomorphic to $\mathbb{R}^{2}$ or off $(\mathbb{R})$.

Assume first that $\mathcal{H}_{2} \cong \mathbb{R}^{2}$. Then, the short exact sequence (9) becomes

$$
0 \rightarrow \mathbb{R} \rightarrow \tilde{\mathcal{G}} \rightarrow \mathbb{R}^{2} \rightarrow 0
$$

Let $\left\{\mathrm{e}_{1}, \mathrm{e}_{2}\right\}$ be a basis for $\mathbb{R}^{2}$. On $\mathbb{R}^{2} \times \mathbb{R}$, the extended Lie bracket given by (4) takes the simplified form

$$
[(x, a),(y, b)]=(0, \phi(x) b-\phi(y) a+\omega(x, y)),
$$

for all $a, b \in \mathbb{R}, x, y \in \mathbb{R}^{2}$.

Setting $\tilde{e}_{i}=\left(e_{i}, 0\right), i=1,2$ and $\tilde{e_{3}}=(0,1)$ we get

$$
\begin{aligned}
& {\left[\tilde{e}_{1}, \tilde{e}_{2}\right]=\omega\left(e_{1}, e_{2}\right) \tilde{e}_{3}} \\
& e_{3}\left[\tilde{e}_{1}, \tilde{e}_{3}\right]=\phi\left(e_{1}\right) \tilde{e}_{3} \\
& {\left[\tilde{e}_{2}, \tilde{e}_{3}\right]=\phi\left(e_{2}\right) \tilde{e}_{3}}
\end{aligned}
$$

Since $\mathcal{G}$ is solvable and non-unimodular, we can, without loss of generality, assume that $\phi\left(e_{2}\right)=0$. That is

$$
D=\left(\begin{array}{ll}
0 & \omega\left(e_{1}, e_{2}\right) \\
0 & \phi\left(e_{1}\right)
\end{array}\right)
$$

Notice that $\phi\left(e_{1}\right)$ should be non- zero, since otherwise $\mathcal{G}$ becomes unimodular. In other words,

$$
D \cong\left(\begin{array}{ll}
0 & 0 \\
0 & 1
\end{array}\right)
$$

Now, we shall determine all the complete left-symmetric structures on $\mathbb{R}^{2}$. These are described by the following lemma that we state without proof.

Lemma 10: Up to left-symmetric isomorphism, there are two complete left-symmetric structures on $\mathbb{R}^{2}$ given, in a basis $\left\{e_{p} e_{2}\right\}$ of $\mathbb{R}^{2}$, by either

(i) $e_{i} \cdot e_{j}=0 \quad \mathrm{i}, \mathrm{j}=1,2$

(ii) $e_{2} \cdot e_{2}=e_{1}$.

From now on, $A_{2}$ will denote the vector space $\mathbb{R}^{2}$ endowed with one of the complete left-symmetric structures described in Lemma 10.

The extended left-symmetric product on $A_{2} \times \mathbb{R}_{0}$ given by (5) turns out to take the simplified form

$$
(x, a) \cdot(y, b)=\left(x \cdot y, b \lambda_{x}+a \rho_{y}+g(x, y)\right),
$$

for all $x, y \in A_{2}$ and $a, b \in \mathbb{R}$. Indeed, $\rho_{x}, \lambda_{x} \in \operatorname{End}(\mathbb{R}) \cong \mathbb{R}$ for all $x \in A_{2}$. So, we can identify $\rho_{x}$ and $\lambda_{x}$ with real numbers that we denote by $\rho_{x}$ and $\lambda_{x}$, respectively. 
Citation: Guediri M, Al-Balawi K (2015) Complete Left-Invariant Affine Structures on Solvable Non-Unimodular Three-Dimensional Lie Groups. J Generalized Lie Theory Appl 9: 222. doi:10.4172/1736-4337.1000222

Page 5 of 11

Note here that $\lambda_{x}=\phi(x)+\rho_{x}$, for all $x \in \mathbb{R}^{2}$ whereas $\phi: \mathbb{R}^{2} \rightarrow \operatorname{End}(\mathbb{R}) \cong \mathbb{R}$ in (10).

The conditions in Theorem 1 can be simplified to the following conditions

$$
\begin{aligned}
& \rho_{(x \cdot y)}=\rho_{y}^{\circ} \rho_{x} \\
& g(x, y \cdot z)-g(y, x . z)+\lambda_{x}(g(y, z))-\lambda_{y}(g(y, z)) \\
& -\rho_{z}(g(x, \mathrm{y})-\mathrm{g}(\mathrm{y}, \mathrm{x}))=0
\end{aligned}
$$

By using (10) and (11), we deduce from

$[(x, a),(y, b)]=(x, a) \cdot(y, b)-(y, b) \cdot(x, a)$,

that

$$
\omega(x, y)=g(x, y)-g(y, x) .
$$

Since $\omega\left(e_{1}, e_{2}\right)=0$, then $g\left(e_{p} e_{2}\right)=g\left(e_{2}, e_{1}\right)$. Since $\phi\left(e_{2}\right)=0$, then $\lambda_{e_{2}}=\rho_{e_{2}}$. Also, since $\phi\left(e_{1}\right) \neq 0$, then $\lambda_{e_{1}}-\rho_{e_{1}} \neq 0$. By applying identity (12) to $e_{i} \cdot e_{i}, \mathrm{i}=1,2$, we deduce that $\rho=0$. Hence $\lambda_{\mathrm{e}_{2}}=0$ and $\lambda_{\mathrm{e}_{1}} \neq 0$, say $\lambda_{\mathrm{e}_{1}}=\alpha, \alpha \in \mathbb{R}^{*}$.

In this case, the formula (6) and (7) become

$$
\delta_{1} h(x, y)=\lambda_{x}(h(y))-h(x \cdot y)
$$

And

$\delta g(x, y, z)=g(x, y \cdot z)-g(y, x \cdot z)+\lambda_{x}(g(y, z))-\lambda_{y}(g(x, z))$

where $h \in \mathcal{L}^{1}\left(A_{2}, \mathbb{R}\right)$ and $g \in \mathcal{L}^{2}\left(A_{2}, \mathbb{R}\right)$.

According to Lemma 10, there are two cases to be considered.

10.1. $A_{2}=\left\langle e_{1}, e_{2}: e_{i} \cdot e_{j}=0, i, j=1,2\right\rangle$.

In this case, using the first formula above for $\delta_{1}$, we get

$$
\delta_{1} h=\left(\begin{array}{cc}
h_{11} & h_{12} \\
0 & 0
\end{array}\right),
$$

Where $h_{11}=\alpha h\left(e_{1}\right)$ and $h_{12}=\alpha h\left(e_{2}\right)$. Similarly, using the second formula above for $\delta_{2}$, we verify easily that if $\mathrm{g}$ is a cocycle (i.e. $\delta_{2} g=0$ ) and $g_{i j}=g\left(e_{i}, e_{j}\right)$, then

$$
g=\left(\begin{array}{cc}
g_{11} & 0 \\
0 & 0
\end{array}\right)
$$

that is $g_{12}=g_{21}=g_{22}=0$. In this case, the class $[g] \in H_{\lambda, \rho}^{2}\left(A_{2}, \mathbb{R}\right)$ of a cocycle $\mathrm{g}$ may be represented, in the basis above, by a matrix of the simplified form

$$
g=\left(\begin{array}{ll}
0 & s \\
0 & 0
\end{array}\right)
$$

We can now determine the extended complete left-symmetric structures on $A_{3}$. By setting $\tilde{e}_{i}=\left(e_{i}, 0\right), i=1,2$ and $\tilde{e}_{3}=(0,1)$ and using formula (11) we obtain that the non- zero relations in $A_{3}$ are

$$
\begin{aligned}
& \tilde{e}_{1} \cdot \tilde{e}_{2}=s \tilde{e}_{3}, \\
& \tilde{e}_{1} \cdot \tilde{e}_{3}=\alpha \tilde{e}_{3},
\end{aligned}
$$$$
\text { with } \alpha=\lambda_{e_{1}} \neq 0
$$

By setting $e_{1}=\frac{1}{\alpha} \tilde{e}_{1}, e_{2}=\tilde{e}_{3}$ and $\tilde{e}_{3}=e_{2}$, and $t=\frac{s}{\alpha}$ we see that the new basis $\left\{e_{p}, e_{2}, e_{3}\right\}$ of $A_{3}$ satisfies

$$
e_{1} \cdot e_{2}=e_{2}
$$$$
e_{l} \cdot e_{3}=t e_{2}
$$

and all other products are zero. We can easily see that this product is isomorphic to

$e_{1} \cdot e_{2}=e_{2}$

We set $N_{3,0}=\left\langle e_{1}, e_{2}, e_{3}: e_{1} \cdot e_{2}=e_{2}\right\rangle$.

10.2. $A_{2}=\left\langle e_{1}, e_{2}: e_{2} \cdot e_{2}=e_{1}\right\rangle$.

We obtain, as above, that $A_{3}$ is isomorphic to one of the following complete left-symmetric algebras

(i) $N_{3,2}=\left\langle e_{1}, e_{2}, e_{3}: e_{1} \cdot e_{2}=e_{2}, e_{3} \cdot e_{3}=e_{1}\right\rangle$,

(ii) $N_{3,3}=\left\langle e_{1}, e_{2}, e_{3}: e_{1} \cdot e_{2}=e_{2}, e_{3} \cdot e_{3}=-e_{1}\right\rangle$.

Assume now that $\mathcal{H}_{2} \cong$ aff $(\mathbb{R})$. Then the extended Lie bracket on aff $(\mathbb{R}) X \mathbb{R}$ given by (4) takes the form

$$
[(x, a),(y, b)]=([x, y], \phi(x) b-\phi(y) a+\omega(x, y)),
$$

for all $a b \in \mathbb{R}, x, y \in \operatorname{aff}(\mathbb{R})$.

Let $\left\{e_{1}, e_{2}\right\}$ be a basis of aff $(\mathbb{R})$ satisfying $\left[e_{1}, e_{2}\right]=e_{2}$. By setting $\tilde{e}_{i}=\left(e_{i}, 0\right), \mathrm{i}=1,2$ and $\tilde{e}_{3}=(0,1)$

we get

$\left[\tilde{e}_{1}, \tilde{e}_{2}\right]=e+\omega\left(e_{1}, e_{2}\right) \tilde{e}_{3}$

$e_{3}\left[\tilde{e}_{1}, \tilde{e}_{3}\right]=\phi\left(e_{1}\right) \tilde{e}_{3}$

$\left[\tilde{e}_{2}, \tilde{e}_{3}\right]=\phi\left(e_{2}\right) \tilde{e}_{3}$

Since $\mathcal{G}$ is solvable and non-unimodular, then as above, we can assume that $\phi\left(e_{2}\right)=0$. That is,

$$
D=\left(\begin{array}{ll}
0 & \omega\left(e_{1}, e_{2}\right) \\
0 & \phi\left(e_{1}\right)
\end{array}\right)
$$

Notice that $\phi\left(e_{1}\right)+1 \neq 0$, since otherwise $\mathrm{g}$ becomes unimodular. Now, we have the following cases.

1. If det $D=0$, then $D \cong\left(\begin{array}{ll}1 & 0 \\ 0 & 1\end{array}\right)$ that is, $\phi\left(e_{1}\right)=0$ and $\omega\left(e_{1}, e_{2}\right)=0$. This means that $\phi$ is identically zero, i.e. $\tilde{\mathcal{G}}$ is a central extension of aff $(\mathbb{R})$ by $\mathbb{R}$.

$$
\text { 2. If } \operatorname{det} D \neq 0, D \cong\left(\begin{array}{ll}
1 & 0 \\
0 & 1
\end{array}\right),\left(\begin{array}{ll}
1 & 1 \\
0 & 1
\end{array}\right) \text { or }\left(\begin{array}{ll}
1 & 0 \\
0 & \mu
\end{array}\right) \text {, with } 0<|\mu|<1 .
$$

It is not hard to prove the following

Lemma 11: Up to left-symmetric isomorphisms, there is a unique complete left-symmetric structure on aff $(\mathbb{R})$ which is given, relative to a basis $e_{p} e_{2}$ of aff $(\mathbb{R})\left[e_{1}, e_{2}\right]=e_{2}$, by $e_{1} \cdot e_{2}=e_{2}$.

We will denote by $N_{2}$ the vector space aff $(\mathbb{R})$ endowed with the complete left-symmetric product given in Lemma 11.

On the other hand, the extended left-symmetric product on $\mathrm{N}_{2} \times$ $\mathbb{R}_{0}$ is given by

$$
(x, a) \cdot(y, b)=(x \cdot y, b \lambda(x)+a \rho(y)+g(x, y)),
$$

for all $a, b \in \mathbb{R}, x, y \in(\mathbb{R})$. 
The conditions in Theorem 1 can be simplified to the following conditions

$$
\begin{aligned}
& \lambda_{[x, y]}=0 \\
& \rho_{(x \cdot y)}=\rho_{y}{ }^{\circ} \rho_{x} \\
& g(x, y \cdot z)-g(y, x \cdot z)+\lambda_{x}(g(y, z))-\lambda_{y}(g(x, z))-g([x, y], z) \\
& -\rho_{z}(g(x, y)-g(y, x))=0
\end{aligned}
$$

By using (10) and (11), we deduce from

$[(x, a),(y, b)]=(x, a) \cdot(y, b)-(y, b) \cdot(x, a)$

that

$$
\omega(x, y)=g(x, y)-g(y, x)
$$

From condition (16), we get $\lambda_{e_{2}}=0$. Applying the identity (17) above to $e_{i} \cdot e_{i}, \mathrm{i}=1,2$, we deduce that $\rho=0$ and hence $\lambda_{e_{1}}=\phi\left(e_{1}\right)$.

Assume first that $D \cong\left(\begin{array}{ll}1 & 0 \\ 0 & 0\end{array}\right)$, that is, $\omega\left(e_{1}, e_{2}\right)=0$ and $\phi\left(e_{1}\right)=0$, then $\lambda=\rho=0$. Thus, the extension is central.

We know that the classification of the exact central extension of $N_{2}$ by $\mathbb{R}_{0}$ is, up to left-symmetric isomorphism, the orbit space of $H_{e x}^{2}\left(N_{2}, \mathbb{R}_{0}\right)$ under the natural action of $G=\operatorname{Aut}\left(\mathbb{R}_{0}\right) \times \operatorname{Aut}\left(N_{2}\right)$ (Proposition3). So, we must compute $H_{e x}^{2}\left(N_{2}, \mathbb{R}_{0}\right)$. Since $\mathbb{R}_{0}$ is a trivial $\mathrm{N}_{2}$-bimodule, then

$$
\begin{aligned}
& \delta_{1} h(x, y)=-h(x \cdot y), \\
& \delta_{2} g(x, y, z)=g(x, y \cdot z)-g(y, x \cdot z)-g([x, y], z),
\end{aligned}
$$

where $h \in \mathcal{L}^{1}\left(N_{2}, \mathbb{R}\right)$ and $g \in \mathcal{L}^{2}\left(N_{2}, \mathbb{R}\right)$. This implies that, with respect to the basis $e_{1}, e_{2}$ of $N_{2}, \delta_{1} h$ is of the form

$$
\delta_{1} h=\left(\begin{array}{cc}
0 & h_{12} \\
0 & 0
\end{array}\right)
$$

where $h_{12}=-h\left(e_{2}\right)$.

Observe that if $\mathrm{g}$ is a 2-cocycle (i.e. $\delta_{2} g=0$ ), then

$$
g=\left(\begin{array}{cc}
g_{11} & 0 \\
0 & 0
\end{array}\right)
$$

where $g_{i j}=g\left(e_{i}, e_{j}\right)$. Hence, $[g] \in H^{2}\left(N_{2}, \mathbb{R}\right)$ can be represented as a matrix with respect to $\left\{e_{1}, e_{2}\right\}$ by

$$
g=\left(\begin{array}{ll}
t & 0 \\
0 & 0
\end{array}\right), t \in \mathbb{R}
$$

We determine, in this case, the extended left-symmetric structure on $A_{3}$. By setting $\tilde{e}_{i}=\left(e_{i}, 0\right), \mathrm{i}=1,2$

and $\tilde{e}_{3}=(0,1)$, and using formula (15), we find

$$
\tilde{e}_{1} \cdot \tilde{e}_{1}=t \tilde{e}_{3}, \quad \tilde{e}_{1} \cdot \tilde{e}_{2}=\tilde{e}_{2} .
$$

and all other products are zero, $t \in \mathbb{R}$. We denote $\mathcal{G}$ endowed with this structure by $N_{3, t}$.

Recall that the extension

$0 \rightarrow \mathbb{R}_{0} \rightarrow A_{3} \rightarrow N_{2} \rightarrow 0$

is exact (i.e. $\left.i\left(\mathbb{R}_{0}\right)=C\left(A_{2}\right)\right)$ if and only if $I_{[g]}=\{0\}$.

Let $x=a e_{1}+b e_{2} \in I_{[g]}$. Then computing all the products $x \cdot e_{i}=e_{i} \cdot x=0$, we deduce that $x=0$, that

is the extension is exact.

Let $N_{3, t}, N_{3, t^{\prime}}$ be two left-symmetric algebras as above. We know that $N_{3, t}$ is isomorphic to $N_{3, t}$ if and only if there exists $(\alpha, \eta) \in \operatorname{Aut}\left(\mathbb{R}_{0}\right) \times \operatorname{Aut}\left(N_{2}\right)=\mathbb{R}^{*} \times \operatorname{Aut}\left(N_{2}\right)$ such that for all $x, y \in N_{2}$, we have

$$
g^{\prime}(x, y)=\alpha g(\eta(x), \eta(y)) .
$$

Now, we have to calculate $\operatorname{Aut}\left(N_{2}\right)$. Let $\eta \in \operatorname{Aut}\left(N_{2}\right)$ so that, with respect to the basis $e_{1}, e_{2}$ of $\mathrm{N}_{2}$ with $e_{1} \cdot e_{2}=e_{2}$,

$$
\eta=\left(\begin{array}{ll}
a & b \\
c & d
\end{array}\right)
$$

Since $\eta\left(e_{2}\right)=\eta\left(e_{1} \cdot e_{2}\right)=\eta\left(e_{1}\right) \cdot \eta\left(e_{2}\right)$, then $b=0$ and $d=a d$. Also $0=\eta\left(e_{1} \cdot e_{1}\right)=\eta\left(e_{1}\right) \cdot \eta\left(e_{1}\right)$ which implies that $a=0$ or $c=0$. Since $\operatorname{det} \eta \neq 0$, then $d \neq 0$ and hence $a=1$ and $c=0$. This means that

$$
\eta=\left(\begin{array}{ll}
1 & 0 \\
0 & d
\end{array}\right)
$$

with $d \neq 0$. We shall now apply formula (18). For this we recall first that in the basis $e_{1}, e_{2}$, the classes $g$ and $g^{\prime}$ corresponding to $N_{3, t}$ and $N_{3, t^{\prime}}$ have, respectively, the forms

$$
g=\left(\begin{array}{ll}
t & 0 \\
0 & 0
\end{array}\right) \text { and } g^{\prime}=\left(\begin{array}{ll}
t^{\prime} & 0 \\
0 & 0
\end{array}\right)
$$

From $g^{\prime}\left(e_{1}, e_{1}\right)=\alpha g\left(\eta\left(e_{1}\right), \eta\left(e_{1}\right)\right)$, we get

$t^{\prime}=\alpha t$

Hence $N_{3, t}$ and $N_{3, t^{\prime}}$ are isomorphic if and only if $t^{\prime}=\alpha t$, for some $\alpha \in \mathbb{R}^{*}$.

Notice that if $t=0$, we obtain the complete left-symmetric algebra $N_{3,0}$ described above. If $t \neq 0$, we obtain, by setting $e_{i}=\tilde{e}_{i}, i=1,2$, and $e_{3}=t \tilde{e}_{3}$, the complete left-symmetric algebra

$$
N_{3,1}=\left\langle e_{1}, e_{2}, e_{3}: e_{1} \cdot e_{1}=e_{3}, e_{1} \cdot e_{2}=e_{2}\right\rangle
$$

Assume now that $D \cong\left(\begin{array}{ll}1 & 0 \\ 0 & 1\end{array}\right)$, that is, $\omega\left(e_{1}, e_{2}\right)=0$ and $\phi\left(e_{1}\right)=1$. Then $\lambda\left(e_{1}\right)=\phi\left(e_{1}\right)=1$. We deduce, in this case, that, in the basis $e_{1}, e_{2}$ of $N_{2}$, the $[g] \in H_{\lambda, \rho}^{2}\left(N_{2}, \mathbb{R}\right)$ of a cocycle $g$ may berepresented by a matrix of the simplified form

$$
g=\left(\begin{array}{ll}
0 & t \\
t & 0
\end{array}\right)
$$

We determine, in this case, the extended complete left-symmetric structure on $A_{3}$. By setting $\tilde{e}_{i}=\left(\tilde{e}_{i}, 0\right), i=1,2$ and $\tilde{e}_{3}=(0,1)$ and using formula (15), we obtain

$$
\begin{aligned}
& \tilde{e}_{1} \cdot \tilde{e}_{2}=\tilde{e}_{2}+t \tilde{e}_{3} \\
& \tilde{e}_{2} \cdot \tilde{e}_{1}=t \tilde{e}_{3} \\
& \tilde{e}_{1} \cdot \tilde{e}_{3}=\tilde{e}_{3}
\end{aligned}
$$

We denote this left-symmetric algebra by $B_{3, t}$. Notice that if $t=0$, we obtain the complete left-symmetric algebra $B_{3,0}$ with the non- zero relations 


$$
e_{1} \cdot e_{2}=e_{2}
$$

$e_{1} \cdot e_{3}=e_{3}$.

If $t \neq 0$; we obtain, by setting $e_{i}=\tilde{e}_{i}, i=1,2$ and $e_{3}=t \tilde{e}_{3}$; the complete left-symmetric algebra $B_{3,1}$ with the non-zero relations

$$
\begin{aligned}
& e_{1} \cdot e_{2}=e_{2}+e_{3} \\
& e_{2} \cdot e_{1}=e_{3} \\
& e_{1} \cdot e_{3}=e_{3}
\end{aligned}
$$

Assume now that $D \cong\left(\begin{array}{ll}1 & 1 \\ 0 & 1\end{array}\right)$ that is, $\omega\left(e_{1}, e_{2}\right)=1$ and $\phi\left(e_{1}\right)=1$. Hence $\lambda\left(e_{1}\right)=\phi\left(e_{1}\right)=1$. Usingthe same method as above, it follows that the class $[g] \in H_{\lambda, \rho}^{2}\left(N_{2}, \mathbb{R}\right)$ of a co-cycle $g$ takes the reduced form

$$
g=\left(\begin{array}{cc}
0 & t \\
t-1 & 0
\end{array}\right)
$$

We determine, in this case, the extended complete left-symmetric structures on $A_{3}$. By setting $\tilde{e}_{i}=\left(e_{i}, 0\right), i=1,2$ and $\tilde{e}_{3}=(0,1)$ and using formula (15), we obtain

$$
\begin{aligned}
& \tilde{e}_{1} \cdot \tilde{e}_{2}=\tilde{e}_{2}+t e_{3} \\
& \tilde{e}_{2} \cdot \tilde{e}_{1}=(t-1) \tilde{e}_{3} \\
& \tilde{e}_{1} \cdot \tilde{e}_{3}=\tilde{e}_{3}
\end{aligned}
$$

We denote such a left-symmetric algebra by $C_{3, t}$. Notice that if $t=1$, we obtain the complete left- symmetric algebra $C_{3,1}$ with the non-zero relations

$$
\begin{aligned}
& e_{1} \cdot e_{2}=e_{2}+e_{3}, \\
& e_{1} \cdot e_{3}=e_{3},
\end{aligned}
$$

and if $t \neq 1$, we obtain the complete left-symmetric algebra $C_{3, \mathrm{t}}$ with the non-zero relations

$$
\begin{aligned}
& e_{1} \cdot e_{2}=e_{2}+t e_{3} \\
& e_{2} \cdot e_{1}=(t-1) e_{3} \\
& e_{1} \cdot e_{3}=e_{3}
\end{aligned}
$$

where different values of $t$ give non-isomorphic complete leftsymmetric algebras.

Assume finally that $D \cong\left(\begin{array}{ll}1 & 0 \\ 0 & \mu\end{array}\right)$, with $0<|\mu|<1$, that is $\omega\left(e_{1}, e_{2}\right)=0$ and $\phi\left(e_{1}\right)=\mu$. Hence $\lambda\left(e_{1}\right)=\phi\left(e_{1}\right)=\mu$. It follows that the class $[g] \in H_{\lambda, \rho}^{2}\left(N_{2}, \mathbb{R}\right)$ of a co-cycle $g$ is identically zero.

We determine, in this case, the extended complete left-symmetric structures on $A_{3}$. By setting $\tilde{e}_{i}=\left(e_{i}, 0\right), i=1,2$ and $\tilde{e}_{3}=(0,1)$ and using formula (15), we obtain

$$
\begin{aligned}
& \tilde{e}_{1} \cdot \tilde{e}_{2}=\tilde{e}_{2}, \\
& \tilde{e}_{1} \cdot \tilde{e}_{3}=\mu \tilde{e}_{3} .
\end{aligned}
$$

where $0<|\mu|<1$. We set

$$
D_{3,1}(\mu)=\left\langle e_{1}, e_{2}, e_{3} e_{1} \cdot e_{2}=e_{2}, e_{1} \cdot e_{3}=\mu e_{3}\right\rangle
$$

where $0<|\mu|<1$.

Case 2: $\mathcal{I} \cong \operatorname{aff}(\mathbb{R})$.

In this case, the short exact sequence (8) becomes

$$
0 \rightarrow N_{2} \rightarrow A_{3} \rightarrow \mathbb{R}_{0} \rightarrow 0
$$

where $N_{2}$ is the complete left-symmetric algebra whose associated Lie algebra is aff $(\mathbb{R})$ and $\mathbb{R}_{0}$ is the trivial left-symmetric algebra over $\mathbb{R}$.

Let $\sigma: \mathbb{R}_{0} \rightarrow A_{3}$ be a section and set $\sigma(1)=x_{0} \in A_{3}$ and define two linear maps $\lambda, \rho \in \operatorname{End}\left(N_{2}\right)$ by putting $\lambda(y)=x_{0} \cdot y$ and $\rho(y)=y \cdot x_{0}$. By setting $e=x_{0} \cdot x_{0}$, we see that $e \in N_{2}$. Let $g: \mathbb{R}_{0} \times \mathbb{R}_{0} \rightarrow N_{2}$ be the bilinear map defined by $g(a, b)=\sigma(a) \cdot \sigma(b)-\sigma(a \cdot b)$. Since the complete leftsymmetric structure on $\mathbb{R}$ is trivial, then $g(a, b)=a b e$, or equivalently $g(1,1)=e$. Also we can show that $\delta_{2} g=0$, i.e. $g \in Z_{\lambda, \rho}^{2}\left(\mathbb{R}_{0}, N_{2}\right)$.

In this case, the extended left-symmetric product on $\mathbb{R}_{0} \oplus N_{2}$ given by (5) takes the simplified form

$$
(a, x) \cdot(b, y)=(0, x \cdot y+a \lambda(y)+b \rho(x)+a b e),
$$

for all $a, b \in \mathbb{R}$ and $x, y \in N_{2}$.

The conditions in Theorem 1 can be simplified to the following conditions

$$
\begin{aligned}
& \lambda(x \cdot y)=\lambda(x) \cdot y+x \cdot \lambda(y)-\rho(x) \cdot y \\
& \rho([x, y])=x \cdot \rho(y)-y \cdot \rho(x) \\
& {[\lambda, \rho]+\rho^{2}=R_{e}}
\end{aligned}
$$

Let $\phi: \mathbb{R} \rightarrow \operatorname{Der}(\operatorname{aff}(\mathbb{R}))$, be a derivation of aff $(\mathbb{R})$. Set

$$
\phi(1)=\left(\begin{array}{ll}
a & c \\
b & d
\end{array}\right)
$$

relative to a basis $e_{1}, e_{2}$ of aff $(\mathbb{R})$ satisfying $\left[e_{1}, e_{2}\right]=e_{2}$. From the identity $\phi(1) e_{2}=\left[\phi(1) e_{1}, e_{2}\right]+\left[e_{1}, \phi(1) e_{2}\right]$, we deduce that $a=c=0$, hence

$$
\begin{aligned}
& \phi(1)=\left(\begin{array}{ll}
0 & 0 \\
b & d
\end{array}\right) \\
& \text { Let } \\
& \rho=\left(\begin{array}{ll}
\alpha_{1} & \beta_{1} \\
\alpha_{2} & \beta_{2}
\end{array}\right)
\end{aligned}
$$

relative to a basis $e_{1}, e_{2}$ of aff $(\mathbb{R})$ satisfying $\left[e_{1}, e_{2}\right]=e_{2}$. Applying formula (21) to $e_{2}$, we get $\beta_{1}=0$. Since $\phi(1)=\lambda-\rho$, we deduce that, relative to the basis $e_{1}, e_{2}$, we have

$$
\lambda=\left(\begin{array}{cc}
\alpha_{1} & 0 \\
\alpha_{2}+b & \beta_{2}+d
\end{array}\right)
$$

Applying formula (20) to all products of the form $e_{i}, e_{j}, i=1$, 2, we get $\alpha_{2}+b=0$. Moreover, by applying formula (22) to $e_{1}$ and $e_{2}$, we get $\alpha_{1}=\beta_{2}=0$. Thus

$$
\rho=\left(\begin{array}{cc}
0 & 0 \\
-b & 0
\end{array}\right) \text { and } \lambda=\left(\begin{array}{ll}
0 & 0 \\
0 & d
\end{array}\right)
$$

Now, since $e \in N_{2}$, then $e=t e_{1}+s e_{2}$ for some $t, s \in \mathbb{R}$. Formula (22) when applied to $e_{1}$ gives

$$
-b d e_{2}=s e_{2}
$$

for which we get that $e=x_{\circ} \cdot x_{\circ}=t e_{1}-b d e_{2}, t \in \dot{\mathbb{R}}$. Hence we get a left-symmetric product on $A_{3}$. Now, let us write down the structure of $A_{3}$ using a basis. From above we have

$$
e_{1} \cdot e_{2}=e_{2}, \quad e_{1} \cdot x_{\circ}=-b e_{2} .
$$




$$
x_{\circ} e_{2}=d e_{2}, \quad x_{\circ} \cdot x_{\circ}=t e_{1}-b d e_{2}, t \in \mathbb{R}
$$

Since $x_{0} \in A_{3}$ and $\pi\left(x_{0}\right)=1$, then $x_{0} \in A_{3} \backslash N_{2}$. Indeed if $x_{0} \in N_{2}$, then the exactness of the short sequence (19) implies that $x_{0} \in i\left(N_{2}\right)=\operatorname{ker} \pi$, a contradiction. This implies that, relative to a basis $\left\{e_{1}, e_{2}, e_{3}\right\}$ of $A_{3}, x_{0}$ is of the form $x_{0}=\alpha e_{1}+\beta e_{2}+\gamma e_{3}$, where $\alpha, \beta, \gamma \in \mathbb{R}$ with $\gamma \neq 0$. In this case, we can, without loss of generality, assume that $\gamma=1$. Thus, $e_{3}=x_{0}-\alpha e_{1}-\beta e_{2}$. Since $e_{1} \cdot x_{\circ}=-b e_{2}$ we get that

$e_{1} \cdot e_{3}=-(b+\beta) e_{2}$,

also since $x_{\circ} \cdot e_{2}=d e_{2}$ we get that

$e_{3} \cdot e_{2}=(d-\alpha) e_{2}$.

since $x_{\circ} \cdot x_{\circ}=t e_{1}-b d e_{2}$, we deduce that

$e_{3} \cdot e_{3}=t e_{1}+(\alpha b+\alpha \beta-b d-\beta d) e_{2}$.

Since $\alpha, \beta$ are arbitrary, we can choose $\alpha, \beta$ so that $e_{3}=x_{\circ}-d e_{1}-b e_{2}$. Hence the left-symmetric product on $A_{3}$ is given, relative the basis $\left\{e_{1}, e_{2}, e_{3}\right\}$, by the non- zero relations

$$
\begin{aligned}
& e_{1} \cdot e_{2}=e_{2} \\
& e_{3} \cdot e_{3}=t e_{1},
\end{aligned}
$$

Notice that if $t=0$, we obtain the complete left-symmetric algebra $N_{3,0}$. If $t \neq 0$, we obtain, by setting $e_{i}=\tilde{e}_{i} ; i=1,2$ and $\tilde{e}_{3}=\frac{1}{\sqrt{|t|}} e_{3}$; that $\mathrm{A}_{3}$ is isomorphic to one of the left-symmetric algebras $N_{3,2}$ or $N_{3,3}$ given above

\section{Case 3: $\mathcal{I} \cong \mathbb{R}^{2}$.}

In this case, the short exact sequence (8) becomes

$$
0 \rightarrow A_{2} \rightarrow A_{3} \rightarrow \mathbb{R}_{0} \rightarrow 0
$$

where $A_{2}$ is a complete left-symmetric algebra whose lie algebra is ${ }^{2}$ and $\mathbb{R}_{0}$ is the trivial left-symmetric algebra over $\mathbb{R}$.

At the lie algebra level, we have a short exact sequence of lie algebras of the form

$$
0 \rightarrow \mathbb{R}^{2} \rightarrow \tilde{\mathcal{G}} \rightarrow \mathbb{R} \rightarrow 0
$$

Let $\phi: \mathbb{R} \rightarrow \operatorname{Der}\left(\mathbb{R}^{2}\right) \cong \operatorname{End}\left(\mathbb{R}^{2}\right)$, be a derivation of $\mathbb{R}^{2}$. Relative to a basis $e_{1}, e_{2}$ of $\mathbb{R}^{2}$ set

$$
\phi(1)=\left(\begin{array}{ll}
a & c \\
b & d
\end{array}\right)
$$

In this case, the extended Lie bracket on $\mathbb{R} \times \mathbb{R}^{2}$, given by (4), takes the simplified form

$$
[(a, x),(b, y)]=(0, \phi(a) y-\phi(b) x+\omega(a, b))
$$

for all $x, y \in \mathbb{R}^{2}$ and $a, b \in \mathbb{R}$. By setting $\tilde{e}_{1}=(1,0)$ and $\tilde{e}_{i+1}=\left(0, e_{i}\right)$, $i=1,2$ we obtain

$$
\begin{aligned}
& {\left[\tilde{\mathrm{e}}_{1}, \tilde{\mathrm{e}}_{2}\right]=\mathrm{a} \tilde{\mathrm{e}}_{1}+\mathrm{b} \tilde{\mathrm{e}}_{2}} \\
& {\left[\tilde{\mathrm{e}}_{1}, \tilde{\mathrm{e}}_{3}\right]=\mathrm{c} \tilde{\mathrm{e}}_{1}+\mathrm{d} \tilde{\mathrm{e}}_{2}} \\
& {\left[\tilde{\mathrm{e}}_{2}, \tilde{\mathrm{e}}_{3}\right]=0}
\end{aligned}
$$

By Lemma 5, we obtain that, relative to the basis $e_{1}, e_{2}$,

$$
D=\left(\begin{array}{ll}
a & b \\
c & d
\end{array}\right)
$$

with $a+d \neq 0$. Note that, in this case, $\omega$ may not be zero, that is, the extensions of $\mathbb{R}$ by $\mathbb{R}^{2}$ are not necessarily semi direct products of $\mathbb{R}$ by $\mathbb{R}^{2}$.

According to Lemma 5, there are five cases to be considered

$$
D \cong\left(\begin{array}{ll}
1 & 0 \\
0 & 0
\end{array}\right),\left(\begin{array}{ll}
1 & 0 \\
0 & 1
\end{array}\right),\left(\begin{array}{ll}
1 & 1 \\
0 & 1
\end{array}\right),\left(\begin{array}{ll}
1 & 0 \\
0 & \mu
\end{array}\right) \text { or }\left(\begin{array}{ll}
1 & -\zeta \\
\zeta & 1
\end{array}\right),
$$

Where $\zeta>0$ and $0<|\mu|<1$.

Let $\sigma: \mathbb{R}_{0} \rightarrow A_{3}$ be a section and set $\sigma(1)=x_{\circ} \in A_{3}$ and define two linear maps $\lambda, \rho \in \operatorname{End}\left(A_{2}\right)$ by putting $\lambda(y)=x_{\circ} \cdot y$ and $\rho(y)=y \cdot x_{\circ}$. By setting $e=x_{\circ} \cdot x_{\circ}$, we see that $e \in A_{2}$. Let $g: \mathbb{R}_{0} \times \mathbb{R}_{0} \rightarrow A_{2}$ be the bilinear map defined by $g(a, b)=\sigma(a) \cdot \sigma(b)-\sigma(a \cdot b)$. Since the complete left-symmetric structure on $\mathbb{R}$ is trivial, then $g(a, b)=a b e$, or equivalently $g(1,1)=e$. Also we can show that $\delta_{2} g=0$, i.e. $g \in Z_{\lambda, \rho}^{2}\left(\mathbb{R}_{0}, A_{2}\right)$.

The extended left-symmetric product on $\mathbb{R}_{0} \oplus A_{2}$ given by (5) is then takes the simplified form

$$
(a, x) \cdot(b, y)=(0, x \cdot y+a \lambda(y)+b \rho(x)+a b e)
$$

for all $x, y \in A_{2}$ and $a, b \in \mathbb{R}$.

The conditions in Theorem 1 can be simplified to the following conditions

$$
\begin{aligned}
& \lambda(x \cdot y)=\lambda(x) \cdot y+x \cdot \lambda(y)-\rho(x) \cdot y \\
& x \cdot \rho(y)-y \cdot \rho(x)=0 \\
& {[\lambda, \rho]+\rho^{2}=R_{e}}
\end{aligned}
$$

According to Lemma 10, we have the following cases of $A_{2}$

1. $A_{2}=\left\langle e_{1}, e_{2}: e_{i} \cdot e_{j}=0, i, j=1,2\right\rangle$.

Assume first that $D \cong\left(\begin{array}{ll}1 & 0 \\ 0 & 0\end{array}\right)$

and let

$$
\rho=\left(\begin{array}{ll}
\alpha_{1} & \beta_{1} \\
\alpha_{2} & \beta_{2}
\end{array}\right)
$$

relative to the basis $e_{1}, e_{2}$ of $A_{2}$. Since $\phi(1)=\lambda-\rho$, we deduce that, relative to the basis $e_{1}, e_{2}$, we have

$$
\lambda=\left(\begin{array}{cc}
\alpha_{1}+1 & \beta_{1} \\
\alpha_{2} & \beta_{2}
\end{array}\right)
$$

Applying formula (26) to $\mathrm{e}_{2}$, we obtain $\beta_{1}=\beta_{2}=0$. The same formula when applied to e yields $\alpha_{1}=\alpha_{2}=0$. It follows that $\rho$ is identically zero and

$$
\lambda=\left(\begin{array}{ll}
1 & 0 \\
0 & 0
\end{array}\right)
$$

We can easily show that the condition (26) above is satisfied for all $e=x_{\circ} \cdot x_{\circ}=s e_{1}+t e_{2}, s t \in \mathbb{R}$. Hence we get a left-symmetric product on $A_{3}$.

Now, let us write down the structure of $A_{3}$ using a basis. From 
above we have

$$
x_{\circ} \cdot e_{1}=e_{1}, \quad x_{\circ} \cdot x_{\circ}=s e_{1}+t e_{2} .
$$

We can easily prove that $x_{0} \in A_{3} \backslash A_{2}$. This implies that, relative to a basis $\left\{e_{1}, e_{2}, e_{3}\right\}$ of $A_{3}, x_{0}$ is of the form $x_{0}=\alpha e_{1}+\beta e_{2}+\gamma e_{3}$, where $\alpha, \beta, \gamma \in \mathbb{R}$ with $\gamma \neq 0$. In this case, we can, without loss of generality, assume that $\gamma=1$. Thus, $e_{3}=x_{0}-\alpha e_{1}-\beta e_{2}$. Since $x_{\circ} \cdot e_{1}=e_{1}$ we get that

$$
e_{3} \cdot e_{1}=e_{1}
$$

also since $x_{\circ} \cdot x_{\circ}=s e_{1}+t e_{2}$, we deduce that

$e_{3} \cdot e_{3}=(s-\alpha) e_{1}+t e_{2}$.

Since $\alpha, \beta$ are arbitrary, we can choose $\alpha, \beta$ so that $e_{3}=x_{\circ}-s e_{1}$. Hence the left-symmetric product on $A_{3}$ is given, relative to the basis $\left\{e_{1}, e_{2}, e_{3}\right\}$ of $A_{3}$, by the non- zero relations

$$
\begin{aligned}
& e_{3} \cdot e_{1}=e_{1} \\
& e_{3} \cdot e_{3}=t e_{2}
\end{aligned}
$$

Notice that if $t=0$, we find the complete left-symmetric algebra $N_{3,0}$. If $t \neq 0$, we get, by setting $\tilde{e}_{1}=e_{3}, \tilde{e}_{2}=e_{1}$, and $\tilde{e}_{3}=t e_{2}$ that $A_{3}$ is isomorphic to the complete left-symmetric algebra $N_{3,1}$.

Assume then that $D \cong\left(\begin{array}{ll}1 & 0 \\ 0 & 1\end{array}\right)$ and let

$\rho=\left(\begin{array}{ll}\alpha_{1} & \beta_{1} \\ \alpha_{2} & \beta_{2}\end{array}\right)$

relative to the basis $e_{1}, e_{2}$ of $\mathrm{A}_{2}$. Since $\phi(1)=\lambda-\rho$, we deduce that, relative to the basis $e_{1}, e_{2}$, we have

$$
\lambda=\left(\begin{array}{cc}
\alpha_{1}+1 & \beta_{1} \\
\alpha_{2} & \beta_{2}+1
\end{array}\right)
$$

By applying formula (26) to $e_{1}$ and $e_{2}$, we get

$$
\begin{aligned}
& \rho=\left(\begin{array}{ll}
0 & \alpha \\
0 & 0
\end{array}\right), \\
& \lambda=\left(\begin{array}{ll}
1 & \alpha \\
0 & 1
\end{array}\right), \alpha \in \mathbb{R}
\end{aligned}
$$

and $e=x_{\circ} \cdot x_{\circ}=\alpha^{2} e_{1}+\alpha e_{2}$.

Similarly, we find that, relative to the basis $\left\{e_{1}, e_{2}, e_{3}\right\}$ of $A_{3}$ with $e_{3}=x_{0}+\alpha^{2} e_{1}-\alpha e_{2}$, the left- symmetric product on $A_{3}$ is given by the non- zero relations

$$
\begin{aligned}
& e_{3} \cdot e_{1}=e_{1} \\
& e_{3} \cdot e_{2}=\alpha e_{1}+e_{2} \\
& e_{2} \cdot e_{3}=\alpha e_{1} .
\end{aligned}
$$

Notice that if $\alpha=0$, we get, by setting $\tilde{e}_{1}=e_{3}, \tilde{e}_{2}=e_{1}$ and $\tilde{e}_{3}=e_{2}$, the complete left-symmetric algebra $B_{3,0}$. If $t \neq 0$ we get, by setting $\tilde{e}_{1}=e_{3} ; \tilde{e}_{2}=e_{2} \tilde{e}_{3}=\alpha e_{1} ;$ that $A_{3}$ is isomorphic to the complete leftsymmetric algebras $B_{3,1}$.

$$
\begin{aligned}
& \text { Assume now that } D \cong\left(\begin{array}{ll}
1 & 1 \\
0 & 1
\end{array}\right), \text { and let } \\
& \rho=\left(\begin{array}{ll}
\alpha_{1} & \beta_{1} \\
\alpha_{2} & \beta_{2}
\end{array}\right)
\end{aligned}
$$

relative to the basis $e_{1}, e_{2}$ of $A_{2}$. Since $D=\lambda-\rho$, we deduce that, relative to the basis $e_{1}, e_{2}$, we have

$$
\lambda=\left(\begin{array}{cc}
\alpha_{1}+1 & \beta_{1}+1 \\
\alpha_{2} & \beta_{2}+1
\end{array}\right)
$$

By applying formula (26) to $e_{1}$ and $e_{2}$, we get

$$
\rho=\left(\begin{array}{ll}
0 & \alpha \\
0 & 0
\end{array}\right), \lambda=\left(\begin{array}{cc}
1 & \alpha+1 \\
0 & 1
\end{array}\right), \alpha \in \mathbb{R}
$$

and $e=x_{\circ} \cdot x_{\circ}=\alpha e_{1}+\alpha e_{2}$.

Similarly, we find that, relative to a basis $\left\{e_{1}, e_{2}, e_{3}\right\}$ of $A_{3}$ with $e_{3}=x_{\circ}+2 \alpha^{2} e_{1}-\alpha e_{2}$, the left-symmetric product on $A_{3}$ is given by the non-zero relations

$$
\begin{aligned}
& e_{3} \cdot e_{1}=e_{1} \\
& e_{3} \cdot e_{2}=(\alpha+1) e_{1}+e_{2} \\
& e_{2} \cdot e_{3}=\alpha e_{1} .
\end{aligned}
$$

Notice that if $\alpha=0$, we get, by setting $\tilde{e}_{1}=e_{3}, \tilde{e}_{2}=e_{1}$ and $\tilde{e}_{3}=e_{2}$ the complete left-symmetric algebra $C_{31}$. If $\alpha \neq 0$, we get, by setting $\alpha=t-1$ with $t \neq 1$, the complete left-symmetric algebra $C_{3,1}$ where different values of $t$ give non-isomorphic complete left-symmetric algebras.

Assume then that $D \cong\left(\begin{array}{ll}1 & 0 \\ 0 & 1\end{array}\right)$, where $0<|\mu|<1$, and let

$$
\rho=\left(\begin{array}{ll}
\alpha_{1} & \beta_{1} \\
\alpha_{2} & \beta_{2}
\end{array}\right)
$$

relative to the basis $e_{1}, e_{2}$ of $\mathrm{A}_{2}$. Since $\phi(1)=\lambda-\rho$, we deduce that, relative to the basis $e_{1}, e_{2}$, we have

$$
\lambda=\left(\begin{array}{cc}
\alpha_{1}+1 & \beta_{1} \\
\alpha_{2} & \beta_{2}+\mu
\end{array}\right)
$$
zero,

By applying formula (26) to $\mathrm{e}_{1}$ and $\mathrm{e}_{2}$, we obtain that $\rho$ is identically

$$
\lambda=\left(\begin{array}{ll}
1 & 0 \\
0 & \mu
\end{array}\right)
$$

and $e=x_{\circ} \cdot x_{\circ}=e_{1}+\mu e_{2}$.

Similarly, we find that, relative to a basis $\left\{e_{1}, e_{2}, e_{3}\right\}$ of $A_{3}$ with $e_{3}=x_{\circ}-e_{1}-e_{2}$, the left-symmetric product on $A_{3}$ is given by the non- zero relations

$$
\begin{aligned}
& e_{3} \cdot e_{1}=e_{1} \\
& e_{3} \cdot e_{2}=\mu e_{2} .
\end{aligned}
$$

By setting $\tilde{e}_{1}=e_{3}, \tilde{e}_{2}=e_{1}$ and $\tilde{e}_{3}=e_{2}$, we get the complete leftsymmetric algebra $D_{3, l}(\mu)$ where $0<|\mu|<1$

Assume finally that $D \cong\left(\begin{array}{ll}1 & -\zeta \\ \zeta & 1\end{array}\right)$, where $\zeta>0$, and let

$$
\rho=\left(\begin{array}{ll}
\alpha_{1} & \beta_{1} \\
\alpha_{2} & \beta_{2}
\end{array}\right)
$$

relative to the basis $e_{1}, e_{2}$ of $A_{2}$. Since $\phi(1)=\lambda-\rho$, we deduce that, relative to the basis $e_{1}, e_{2}$ above, we have

$$
\lambda=\left(\begin{array}{cc}
\alpha_{1}+1 & \beta_{1}-\zeta \\
\alpha_{2}+\zeta & \beta_{2}+1
\end{array}\right)
$$


Citation: Guediri M, Al-Balawi K (2015) Complete Left-Invariant Affine Structures on Solvable Non-Unimodular Three-Dimensional Lie Groups. J Generalized Lie Theory Appl 9: 222. doi:10.4172/1736-4337.1000222

Page 10 of 11 zero,

By applying formula (26) to $e_{1}$ and $e_{2}$, we obtain that $\rho$ is identically

$$
\lambda=\left(\begin{array}{cc}
1 & -\zeta \\
\zeta & 1
\end{array}\right)
$$

and $e=x_{\circ} \cdot x_{\circ}=2 \zeta e_{1}+\left(\zeta^{2}-1\right) e_{2}$.

Similarly, we find that, relative to a basis $\left\{e_{1}, e_{2}, e_{3}\right\}$ of $A_{3}$ with $e_{3}=x_{\circ}-\zeta e_{1}+e_{2}$, the left-symmetric product on $A_{3}$ is given by the non- zero relations

$$
\begin{aligned}
& e_{3} \cdot e_{1}=e_{1}+\zeta e_{2} \\
& e_{3} \cdot e_{2}=-\zeta e_{1}+e_{2}
\end{aligned}
$$

Set $\tilde{e}_{1}=e_{3}, \tilde{e}_{2}=e_{1}$ and $\tilde{e}_{3}=e_{2}$. Then, the non- zero relations above become

$$
\begin{aligned}
& \tilde{e}_{1} \cdot \tilde{e}_{2}=\tilde{e}_{2}+\zeta \tilde{e}_{3} \\
& \tilde{e}_{1} \cdot \tilde{e}_{3}=-\zeta \tilde{e}_{2}+\tilde{e}_{3}
\end{aligned}
$$

We set

$$
\left.E_{3, \zeta}=\left\langle e_{1}, e_{2}, e_{3}: e_{1} \cdot e_{2}=e_{2}+\zeta e_{3}, e_{1} \cdot e_{3}=-\zeta e_{2}+e_{3}, \zeta\right\rangle 0\right\rangle .
$$$$
\text { 2. } A_{2}=\left\langle e_{1}, e_{2}: e_{2} \cdot e_{2}=e_{1}\right\rangle \text {. }
$$

Let

$$
\rho=\left(\begin{array}{ll}
\alpha_{1} & \beta_{1} \\
\alpha_{2} & \beta_{2}
\end{array}\right)
$$

relative to the basis $e_{1}, e_{2}$ of $A_{2}$. By applying formula (25) to $e_{1}$ and $e_{2}$, we get that $\alpha_{2}=0$

Assume first that $D \cong\left(\begin{array}{ll}1 & 0 \\ 0 & 0\end{array}\right)$. Then, as $\phi(1)=\lambda-\rho$, we deduce that, relative to the basis $e_{1}, e_{2}$, we have

$$
\lambda=\left(\begin{array}{cc}
\alpha_{1}+1 & \beta_{1} \\
0 & \beta_{2}
\end{array}\right)
$$

By applying formula (26) to $e_{1}$ and $e_{2}$, we get that $\alpha_{1}=\beta_{2}=0$. Moreover, by applying formula (24) to all products of the form $e_{i} \cdot e_{j}, i, j=1,2$, we get that $1=0$, a contradiction. Thus $D$ cannot be of this form. Similarly, we can prove that $D$ cannot be of the forms $\left(\begin{array}{ll}1 & 0 \\ 0 & 1\end{array}\right),\left(\begin{array}{ll}1 & 1 \\ 0 & 1\end{array}\right), \operatorname{or}\left(\begin{array}{ll}1 & -\zeta \\ \zeta & 1\end{array}\right), \zeta>0$.

where $\zeta>0$.

Assume that $D \cong\left(\begin{array}{ll}1 & 0 \\ 0 & \mu\end{array}\right)$, where $0<|\mu|<1$, Then, as $\phi(1)=\lambda-\rho$, we deduce that

$$
\lambda=\left(\begin{array}{cc}
\alpha_{1}+1 & \beta_{1} \\
0 & \beta_{2}+\mu
\end{array}\right)
$$

By applying formula (26) to $e_{1}$ and $e_{2}$, we get that $\alpha_{1}=\beta_{2}=0$. Moreover, by applying formula (24) to all products of the form $e_{i} \cdot e_{j}, i, j=1,2$, we get that $\mu=\frac{1}{2}$. Thus

$$
\begin{aligned}
& \rho=\left(\begin{array}{ll}
0 & \alpha \\
0 & 0
\end{array}\right), \lambda=\left(\begin{array}{ll}
1 & \alpha \\
0 & \frac{1}{2}
\end{array}\right), \alpha \in \mathbb{R} \\
& \text { and } e=x_{\circ} \cdot x_{\circ}=t e_{1}+\frac{1}{2} \alpha e_{2}, t \in \mathbb{R} .
\end{aligned}
$$

Similarly, we find that, relative to a basis $\left\{e_{1}, e_{2}, e_{3}\right\}$ of $A_{3}$ with $e_{3}=x_{\circ}+\left(\alpha^{2}-t\right) e_{1}-\alpha e_{2}$, the left-symmetric product on $A_{3}$ is given by the non-zero relations

$$
\begin{aligned}
& e_{2} \cdot e_{2}=e_{1}, \\
& e_{3} \cdot e_{1}=e_{1}, \\
& e_{3} \cdot e_{2}=\frac{1}{2} e_{2},
\end{aligned}
$$
become

Set $\tilde{e}_{1}=e_{3}, \tilde{e}_{2}=e_{1}$ and $\tilde{e}_{3}=e_{2}$. Then the non- zero relations above

$$
\begin{aligned}
& \tilde{e}_{2} \cdot \tilde{e}_{2}=\tilde{e}_{1}, \\
& \tilde{e}_{1} \cdot \tilde{e}_{2}=\tilde{e}_{2}, \\
& \tilde{e}_{1} \cdot \tilde{e}_{3}=\frac{1}{2} \tilde{e}_{3}
\end{aligned}
$$

We set

$$
D_{3,2}=\left\langle e_{1}, e_{2}, e_{3}: e_{2} \cdot e_{2}=e_{1}, e_{1} \cdot e_{2}=e_{2}, e_{1} \cdot e_{3}=\frac{1}{2} e_{3}\right\rangle \text {. }
$$

\section{Conclusion}

We can now state the main result of this paper

Theorem 12: Let $A_{3}$ be a three dimensional complete left-symmetric algebra whose associated Lie algebra $\mathcal{G}$ is solvable and non-unimodular. Then $\mathrm{A}_{3}$ is isomorphic to one of the following left-symmetric algebras (Table 1).

\begin{tabular}{|l|l|c|c|}
\hline Name & Non-zero product & Lie algebra & Remarks \\
\hline$N_{3,0}$ & $e_{1} \cdot e_{2}=e_{2}$ & $\mathcal{G}_{3,1}$ & $N, D, S$ \\
\hline$N_{3,1}$ & $e_{1} \cdot e_{1}=e_{3}, e_{1} \cdot e_{2}=e_{2}$ & $\mathcal{G}_{3,1}$ & $N, D, S$ \\
\hline$N_{3,2}$ & $e_{1} \cdot e_{2}=e_{2}, e_{3} \cdot e_{3}=e_{1}$ & $\mathcal{G}_{3,1}$ & $S$ \\
\hline$N_{3,3}$ & $e_{1} \cdot e_{2}=e_{2}, e_{3} \cdot e_{3}=-e_{1}$ & $\mathcal{G}_{3,1}$ & $S$ \\
\hline$B_{3,0}$ & $e_{1} \cdot e_{2}=e_{2}, e_{1} \cdot e_{3}=e_{3}$ & $\mathcal{G}_{3,2}$ & $N, D, S$ \\
\hline$B_{3,1}$ & $\begin{array}{c}e_{1} \cdot e_{2}=e_{2}+e_{3}, \\
e_{2} \cdot e_{1}=e_{3}, e_{1} \cdot e_{3}=e_{3}\end{array}$ & $\mathcal{G}_{3,2}$ & $D$ \\
\hline$C_{3,1}$ & $\begin{array}{l}e_{1} \cdot e_{2}=e_{2}+e_{3}, \\
e_{1} \cdot e_{3}=e_{3}\end{array}$ & $\mathcal{G}_{3,3}$ & $N, D, S$ \\
\hline$C_{3, t}$ & $e_{1} \cdot e_{2}=e_{2}+t e_{3}, e_{1} \cdot e_{3}=e_{3}$, & $\mathcal{G}_{3,3}$ & $D$ \\
\hline$D_{3,1}(\mu)$ & $e_{2} \cdot e_{1}=(t-1) e_{3}, t \neq 1$ & & \\
\hline$D_{3,2}$ & $e_{1} \cdot e_{2}=e_{2}$, & $\mathcal{G}_{3,4}^{\mu}$ & $N, D, S$ \\
& $e_{1} \cdot e_{3}=\mu e_{3}, 0<|\mu|<1$ & & \\
\hline$E_{3,1}(\zeta)$ & $e_{1} \cdot e_{2}=e_{2}, e_{1} \cdot e_{3}=\frac{1}{2} e_{3}$, & $\mathcal{G}_{3,4}^{\frac{1}{2}}$ & \\
& $e_{2} \cdot e_{2}=e_{1}$ & & \\
\hline & $e_{1} \cdot e_{2}=e_{2}+\zeta e_{3}$, & $\mathcal{G}_{3,5}^{\zeta}$ & $N, D, S$ \\
\hline & $e_{3}=-\zeta e_{2}+e_{3}, \zeta>0$ & & \\
\hline
\end{tabular}

Table 1: Left-symmetric algebras.

Here, the letter $N$ that the left-symmetric algebra $A_{3}$ is Novikov, the letter $D$ means that $A_{3}$ is derivation and the letter $S$ means that $A_{3}$ satisfying $[x, y] \cdot z=0$ for all $x, y, z \in A_{3}$.

Remark 1: We note that left-symmetric algebras satisfying the identity $(x \cdot y) \cdot z=(y \cdot x) \cdot z$ for all $x, y, z \in A$ (or equivalently, the 
Citation: Guediri M, Al-Balawi K (2015) Complete Left-Invariant Affine Structures on Solvable Non-Unimodular Three-Dimensional Lie Groups. J Generalized Lie Theory Appl 9: 222. doi:10.4172/1736-4337.1000222

identity $[x, y] \cdot z=0$ for all $x, y, z \in A$ are of special interest because they correspond to locally simply transitive at ne actions of Lie groups $G$ on a vector space E such that the commutator subgroup $[G, G]$ is acting by translations. These left-symmetric algebras have been considered and studied in [7].

We note that the mapping $X \rightarrow\left(L_{X}, X\right)$ is a Lie algebra representation of $\mathcal{G}$ in aff $\left(\mathbb{R}^{3}\right)=\operatorname{End}\left(\mathbb{R}^{3}\right) \oplus \mathbb{R}^{3}$.

By using the exponential maps, Theorem 12 can now be stated, in terms of simply transitive actions of subgroups of the affine group $\operatorname{Aff}\left(\mathbb{R}^{3}\right)=G L\left(\mathbb{R}^{3}\right) \mathbb{R}^{3}$, as follows

To state it, define the continuous functions $f, g, h, k$ and $\phi$ by

$$
\begin{aligned}
& f(x)=\left\{\begin{array}{ll}
\frac{e^{x}-1}{x}, & x \neq 0 \\
1 & x=0
\end{array}, g(x)=\left\{\begin{array}{ll}
\frac{e^{x}-x-1}{x^{2}}, & x \neq 0 \\
\frac{1}{2} & x=0
\end{array},\right.\right. \\
& h(x)=\left\{\begin{array}{ll}
\frac{\cos x-1}{x}+\frac{x}{2}, & x \neq 0 \\
0 & x=0
\end{array}, k(x)=\left\{\begin{array}{ll}
\frac{\sin x-x}{x}, & x \neq 0 \\
0 & x=0
\end{array},\right.\right. \\
& \phi(x)=\sum_{n=1}^{\infty} \frac{n x^{n}}{(n+1) !}
\end{aligned}
$$

Theorem 13: Suppose that the Lie group $G$ of the non-unimodular Lie algebra $\mathcal{G}$ of dimension 3 acts simply transitively by affine transformations on $\mathbb{R}^{3}$. Then, as a subgroup of $A f f\left(\mathbb{R}^{3}\right), G$ is conjugate to one of the following sub groups:

$$
\begin{aligned}
& G_{A_{3,0}}=\left\{\left(\begin{array}{lll}
1 & 0 & 0 \\
0 & e^{a} & 0 \\
0 & 0 & 1
\end{array}\right)\left[\begin{array}{l}
a \\
b f(a) \\
c
\end{array}\right], a, b, c \in \mathbb{R}\right\} \\
& G_{A_{3,1}}=\left\{\left(\begin{array}{lll}
1 & 0 & 0 \\
0 & e^{a} & 0 \\
a & 0 & 1
\end{array}\right)\left[\begin{array}{l}
a \\
b f(a) \\
c+\frac{1}{2} a^{2}
\end{array}\right], a, b, c \in \mathbb{R}\right\} \\
& G_{A_{3,2}}=\left\{\left(\begin{array}{lll}
1 & 0 & c \\
0 & e^{a} & 0 \\
0 & 0 & 1
\end{array}\right)\left[\begin{array}{l}
a+\frac{1}{2} c^{2} \\
b f(a) \\
c
\end{array}\right], a, b, c \in \mathbb{R}\right\} \\
& G_{A_{3,3}}=\left\{\left(\begin{array}{ccc}
1 & 0 & -c \\
0 & e^{a} & 0 \\
0 & 0 & 1
\end{array}\right)\left[\begin{array}{l}
a-\frac{1}{2} c^{2} \\
b f(a) \\
c
\end{array}\right], a, b, c \in \mathbb{R}\right\} \\
& G_{B_{3,0}}=\left\{\left(\begin{array}{lll}
1 & 0 & 0 \\
0 & e^{a} & 0 \\
0 & 0 & e^{a}
\end{array}\right)\left[\begin{array}{l}
a \\
b f(a) \\
c f(a)
\end{array}\right], a, b, c \in \mathbb{R}\right\} \\
& G_{B_{3,1}}=\left\{\left(\begin{array}{lll}
1 & 0 & 0 \\
0 & e^{a} & 0 \\
b f(a) & a e^{a} & e^{a}
\end{array}\right)\left[\begin{array}{l}
a \\
b f(a) \\
(a b+c) f(a)
\end{array}\right], a, b, c \in \mathbb{R}\right\}
\end{aligned}
$$

$$
\begin{aligned}
G_{C_{3,1}} & =\left\{\left(\begin{array}{lll}
1 & 0 & 0 \\
0 & e^{a} & 0 \\
0 & a e^{a} & e^{a}
\end{array}\right)\left[\begin{array}{l}
a \\
b f(a) \\
c f(a)+b \phi(a)
\end{array}\right], a, b, c \in \mathbb{R}\right\} \\
G_{C_{3, t}} & =\left\{\left(\begin{array}{lll}
1 & 0 & 0 \\
0 & e^{a} & 0 \\
(t-1) b f(a) & t a e^{a} & e^{a}
\end{array}\right)\left[\begin{array}{l}
a \\
b f(a) \\
(t a b+c-b) f(a)+b
\end{array}\right], a, b, c \in \mathbb{R}, t \neq 1\right\}
\end{aligned}
$$$$
G_{D_{3,1}(\mu)}=\left\{\left(\begin{array}{lll}
1 & 0 & 0 \\
0 & e^{a} & 0 \\
0 & 0 & e^{\mu a}
\end{array}\right)\left[\begin{array}{l}
a \\
b f(a) \\
c f(\mu a)
\end{array}\right], a, b, c \in \mathbb{R}, 0<|\mu|<1\right\}
$$$$
G_{D_{3,2}}=\left\{\left(\begin{array}{lll}
1 & b f(a) & 0 \\
0 & e^{a} & 0 \\
0 & 0 & e^{\frac{1}{2} a}
\end{array}\right)\left[\begin{array}{l}
a+b^{2} g(a) \\
b f(a) \\
c f\left(\frac{a}{2}\right)
\end{array}\right], a, b, c \in \mathbb{R}\right\}
$$$$
G_{E_{3}(\zeta)}=\left\{\begin{array}{l}
\left(\begin{array}{lll}
1 & 0 & 0 \\
0 & e^{a} \cos \zeta a & -e^{a} \sin \zeta a \\
0 & e^{a} \sin \zeta a & e^{a} \cos \zeta a
\end{array}\right) \\
{\left[\begin{array}{ll}
a & \\
b(f(a)+k(\zeta a))+c(h(\zeta a)-\zeta \phi(a)) \\
b(\zeta \phi(a)-h(\zeta a))+c(f(a)+k(\zeta a))
\end{array}\right], a, b, c \in \mathbb{R}, \zeta>0}
\end{array}\right\}
$$

\section{Acknowledgment}

The first author would like to extend his sincere appreciation to the Deanship of Scientific Research at King Saud University for its funding of this research through the Research Group No. RGP-1435-069.

\section{References}

1. Koszul JL (1961) Domaines bornes homogenes et orbites de groups de transformations affines. Bull Soc Math France 89: 515-533.

2. Vinberg E (1963) The theory of convex homogeneous cones. Transl Moscow Math Soc 12: 303-358.

3. Milnor J (1977) On fundamental groups of complete affinity flat manifolds. Adv Matt 25: 178-187.

4. Segal D (1992) The structure of complete left-symmetric algebras. Math Ann 293: $569-578$

5. Kim H (1986) Complete left-invariant affine structures on nilpotent lie groups Diff Geom 24: 373-394.

6. Auslander L (1977) Simply transitive groups of affine motions. Amer Math 99 809-826.

7. Benoist $Y(1995)$ Une nilvariete non affine. J Differential Geom 41: 21-52.

8. Fried D, Goldman W (1983) Three dimensional affine crystallographic groups Advances in Math 47: 1-49.

9. Kim H (1986) Complete left-invariant affine structures on nilpotent Lie groups Diff Geom 24: 373-394.

10. Guediri M (2014) Classification of complete left-invariant affine structures on the oscillator group. Math Commun 19: 343-362.

11. Burde D (1998) Simple left-symmetric algebras with solvable lie algebra Manuscript Math 95: 397-411.

12. Kong X, Bai CM, Meng D (2012) On real simple left-symmetric algebras. Comm in Algebra 40: 1641-1668.

13. Neeb KH (2006) Non-abelian extensions of topological lie algebras. Comm in Algebra 34: 991-1041.

14. Jacobson N (1979) Lie algebras. Dover Publications, New York

15. Chang K, Kim H, Lee H (2004) Radicals of a left-symmetric algebra on a nilpotent lie group. Bull Korean Math Soc 41: 359-369.

16. Milnor J (1976) Curvatures of left invariant metrics on lie groups. Advances in Math 21: 293-329. 